AsCLEPIO. Revista de Historia de la Medicina y de la Ciencia

69 (2), julio-diciembre 2017, p189

ISSN-L:0210-4466

http://dx.doi.org/10.3989/asclepio.2017.12

DOSSIER: ESCENARIOS DE LAS PRÁCTICAS PSIQUIÁTRICAS EN AMÉRICA LATINA (SIGLOS XIX Y XX) / OVERVIEW OF PSYCHIATRIC PRACTICES IN LATIN AMERICA (1 $9^{\text {th }}$ AND $20^{\text {th }}$ CENTURIES)

\title{
LA ANATOMOPATOLOGÍA ALEMANA EN EL CENTRO DE LA PSIQUIATRÍA ARGENTINA. UNA APROXIMACIÓN A LOS ESTUDIOS CLÍNICOS EN EL HOSPICIO DE LAS MERCEDES (1900-1910)
}

\author{
Aida Alejandra Golcman \\ CONICET-Universidad Nacional de Tucumán \\ alejandragolcman@gmail.com \\ ORCID iD: http://orcid.org/0000-0002-6609-5749 \\ Marco Antonio Ramos \\ Yale University \\ marco.ramos@yale.edu \\ ORCID iD: http://orcid.org/0000-0002-9394-2763
}

Recibido: 16 abril 2017; Aceptado: 25 junio 2017.

Cómo citar este artículo/Citation: Golcman, Aida Alejandra y Ramos, Marco Antonio (2017), "La anatomopatología alemana en el centro de la psiquiatría argentina. Una aproximación a los estudios clínicos en el Hospicio de las Mercedes (1900-1910)", Asclepio 69 (2): p189. doi: http://dx.doi.org/10.3989/asclepio.2017.12

RESUMEN: En este trabajo nos ocupamos del desarrollo de la anatomopatología en el campo psiquiátrico en Buenos Aires, Argentina, principalmente en el Hospicio de las Mercedes a principios del 1900. Por medio del doctor Domingo Cabred, el gobierno argentino contrató al médico alemán Cristofredo Jacob a través del Ministerio de Relaciones Exteriores, para que se hiciera cargo del Laboratorio de Clínica Psiquiátrica y Neurológica del Hospicio de las Mercedes (1889-1904). Para facilitar su trabajo, se construyó un laboratorio que era la réplica exacta del laboratorio de anatomía patológica en el que Jacob trabajaba en Alemania. Su labor fue fundamental, dado que cimentó la escuela neurobiológica argentina, campo en el que dejó importantes discípulos (Stagnaro, 2006). A partir de este contexto, investigaremos en este artículo parte del trabajo desarrollado en dicho laboratorio. Nos centraremos en los registros de una población de pacientes que fallecieron y fueron autopsiados en el hospicio. Examinaremos cuáles fueron los diagnósticos y analizaremos algunos vínculos entre la clínica de investigación y la teoría de la época referida a la anatomía patológica. Demostraremos que las prácticas del laboratorio -no sólo la autopsia, sino también los análisis de sangre- eran tecnologías críticas para sostener el marco diagnóstico higiénico. Las autopsias construyeron la conexión causal entre los cambios del cuerpo anatomoclínico y la psicopatología; y así, establecía la disciplina psiquiátrica como una rama legitima de la medicina, que generaba explicaciones científicas para la problemática social de la inmigración en el cambio de siglo en Argentina.

PALABRAS CLAVE: Psiquiatría; Anatomía; Neurobiología; Argentina; Domingo Cabred; Hospicio de las Mercedes.

\section{GERMAN ANATOMICAL PATHOLOGY IN THE CENTER OF ARGENTINE PSYCHIATRY: CLINICAL STUDIES AT THE MERCEDES HOSPITAL (1900-1910)}

ABSTRACT: In this article, we explore the development of pathological anatomy in the psychiatric field in Buenos Aires, Argentina, particularly in the Mercedes hospital beginning around 1900. The Argentine government, by means of Dr. Domingo Cabred, contracted German physician Christofried Jakob, through the Ministry of Foreign Relations, to take charge of the Laboratory of Clinical Psychiatry and Neurology at the Mercedes Hospital (1889-1904). To facilitate this work, a laboratory was constructed that was an exact replica of the laboratory of pathological anatomy developed in Germany. This work was critical to cementing the neurobiological school in Argentina and produced important followers of the biological approach to psychiatry. In this article, we investigate a part of the work that developed in this laboratory. Specifically, we focus on the registry of a population of patients who died and were subsequently autopsied in the hospital. We examine the diagnoses that were used to characterize this population and analyze the relationship between laboratory practice and the theory of anatomical pathology during this period. We show that laboratory practices - not just autopsy but also blood tests - were critical technologies to sustain a hygienic diagnostic framework in the hospital. Autopsies demonstrated the causal connection between changes in the anatomical-clinical body and psychopathology and thus, established the psychiatric field as a legitimate branch of medicine that produced scientific explanations for the social problematic of immigration at the turn of the century in Argentina.

KEY WORDS: Psychiatry; Anatomy; Neurobiology; Argentina; Domingo Cabred; Mercedes Hospital.

Copyright: ( 2017 CSIC. Este es un artículo de acceso abierto distribuido bajo los términos de la licencia Creative Commons Attribution (CC BY) España 3.0. 


\section{INTRODUCCIÓN}

En este trabajo nos ocupamos del desarrollo de la anatomopatología en Buenos Aires, Argentina, principalmente en el Hospicio de las Mercedes, a principios del 1900. Esta corriente médica de investigación, que llegó a la Argentina entre las últimas décadas del siglo XIX y las primeras del siglo XX, se ubicó en las filas del materialismo. El pensamiento psiquiátrico argentino llegó a la corriente materialista impulsado por el positivismo básico, de acuerdo con el cual se requiere de la comprobación verificada de los sentidos para respaldar el conocimiento. A partir de este contexto, en este artículo investigaremos parte del trabajo desarrollado en un laboratorio de anatomía patológica de dicho hospital. Nos centraremos en los registros de una población de pacientes que fallecieron en él y analizaremos algunas vinculaciones entre la clínica de investigación y la teoría de la época referida a la anatomía patológica y a las concepciones psiquiátricas en general.

En la cultura científica en Argentina de fin de siglo XIX, el positivismo y el modernismo cultural se disputaban con otras expresiones la construcción de ideas sobre la nación y la sociedad (Terán, 2000, pp. 327364; Terán, 2004, pp. 13-97). Los pensadores de la época se preocuparon por la vida en las ciudades, y por lo que se consideró como los efectos indeseados de la modernidad, por ejemplo, los personajes problemáticos que derivaban de ella: vagos, mendigos, locos y delincuentes. Este problema común a toda la modernidad adoptaba rasgos particulares en una Argentina en proceso de recepción de inmigrantes europeos y que venía de un régimen elitista, en un sistema político excluyente (Terán, 2000, pp. 333-335). Además, la historiografía especializada subraya que, mientras en América Latina se destacaban las ideas positivistas, en Europa, estas ya estaban siendo impugnadas por corrientes como el vitalismo (Roig, 1963, pp. 159-182). La sociedad y sus problemáticas eran interpretadas a partir del determinismo biológico y se legitimaban en las ciencias naturales. Se sostenía la existencia de una minoría que contaba con un capital intelectual superior, que debía ocuparse de entender a la sociedad y de marcar el recorrido de las políticas a desarrollar (Terán, 1987, pp. 46-47). ${ }^{1}$

La historiografía destaca diversos personajes relevantes de la cultura argentina de la época que respondieron a esta línea positivista, en la que se encuadra el discurso de José Ingenieros como el más difundido en la Argentina. Dicho autor utilizó categorías pertenecientes a una "sociología científica" que respondían al positivismo evolucionista y al darwinismo, y en cierto momento de su producción vinculó sus ideas con las del italiano Cesare Lombroso (Terán, 2000 , p. 332). Consideraba necesarias a las ciencias sociales para normatizar la segregación de los grupos patologizados que no se integraban al ideal de búsqueda por una nación moderna (Terán, 1987, p. 49). En su discurso, sostuvo que era necesario clasificar y dividir a los individuos, y que las terapéuticas necesarias para atacar la enfermedad social en cada caso correspondían a las características de los sujetos sociales que las padecían (Terán, 1987, p. 46). Mencionamos superficialmente las ideas de este actor clave en la intelectualidad argentina finisecular, como ejemplo para graficar el marco de pensamiento en el que se practicó la anatomía patológica en el Hospicio de las Mercedes. El trabajo clínico y a la vez de control social que desempeñaron los psiquiatras de la época implicó una combinación del ámbito jurídico y el médico que pretendió ordenar la vida cotidiana y segregar al diferente; por lo tanto, el rol del médico ocupó un lugar más central en la vida social, y también en las instituciones como asilos, hospitales generales y cárceles.

La historiografía de la psiquiatría argentina basó sus trabajos sobre esta temática en la figura del médico alemán Cristofredo Jacob (1866-1956) (Golcman, 2015a, p. 167), ${ }^{2}$ quien fuera contratado por el gobierno argentino por medio del doctor Domingo Cabred y a través del Ministerio de Relaciones Exteriores para hacerse cargo del Laboratorio de Clínica Psiquiátrica y Neurológica del Hospicio de las Mercedes durante la presidencia de Julio Roca. Para facilitar su trabajo, se construyó una réplica exacta del laboratorio de anatomía patológica en el que Jacob trabajaba en Alemania. Su labor fue fundamental, dado que, entre otros hitos, cimentó la escuela neurobiológica argentina, donde dejó importantes discípulos. Además, a partir de la presencia de Jacob, se instalaron en la Argentina las concepciones de la escuela alemana de neuropsiquiatría que se inscribían en el pensamiento de Carl Wernicke (Stagnaro, 2006). ${ }^{3}$

Uno de sus discípulos fue José Tiburcio Borda (1869-1936), quien había comenzado a trabajar en el Hospicio de las Mercedes en 1895, fue titular de la cátedra de Clínica Psiquiátrica entre 1922 y 1930 y, a partir de ese año, miembro titular de la Academia de Medicina. La institución que fuera el hospicio lleva su nombre desde 1967. Sus trabajos de investigación en anatomía patológica le significaron un reconocimiento internacional. Borda fue uno de los persona- 
jes destacados que escribieron sobre esta temática, e integró a su experiencia clínica las teorías de Valentin Magnan, Emmanuel Regis y Emil Kraepelin (Golcman, 2015b, p. 168).

\section{CONTEXTO HOSPITALARIO DEL LABORATORIO DE ANATOMÍA PATOLÓGICA}

El positivismo inspiró un Estado normatizador que se ocupó de varios frentes: penitenciario, judicial, sanitario, educativo, etc. Con estas reformas institucionales se pretendía controlar y modificar la conducta de los sujetos subalternos para acercarla al modelo de ciudadano planteado por el positivismo (Bohoslavsky, 2005). Claro está, los autores especializados se han encargado de mostrar los matices propios de cada institución, y los espacios de negociación y participación de los sujetos que eran parte de dichas instituciones (Bohoslavsky, 2005, p. 50).

La historiografía comparte la idea de que, con la consolidación nacional y el establecimiento de un gobierno constitucional, en la década de 1880 hubo un profundo cambio en el tratamiento de la patología mental. Se generó un dispositivo psiquiátrico, con la presencia de los hospicios, la generación de algunas cátedras y la producción de ciertas publicaciones (Golcman, 2015a, p. 61; Vezzetti, 1985, p. 49). En las tres décadas que siguieron, el Estado argentino reformó los asilos existentes y desarrolló una red de instituciones modernas diseñadas para tratar la locura (Ablard, 2008, p. 17).

Estos hechos sucedieron en el marco del higienismo de fines del siglo XIX, que formó parte de un discurso sobre el progreso y la civilización (Vezzetti, 1985, p. 37). En esta corriente de pensamiento del siglo XIX, la enfermedad era entendida como un fenómeno social y político que debía permanecer bajo la autoridad y tutela del Estado. En este marco de ideas se insertaron las prácticas psiquiátricas dentro de las instituciones hospitalarias, y la medicina y la política se vincularon a partir de cuestiones técnicas y morales. La sociedad fue entendida a la manera de un organismo, y los médicos ocuparon un lugar relevante para tratar sus problemas, ya que los conflictos sociales se concebían como patologías, y se sostuvo que las prácticas sociales "viciosas" (por ejemplo, locura, anarquismo, vagancia, miseria) producían la degeneración. La psiquiatría ocupó un lugar entre las disciplinas que se desarrollaron para encontrar soluciones a estas problemáticas (Talak, 2005, pp. 563-599).

El Hospicio de las Mercedes se inauguró el 11 de noviembre de 1863 . Su primer director fue el Dr. José
María Uriarte, sucedido en 1876 por el Dr. Lucio Meléndez, quien fue en alguna medida la cara visible de la institucionalización psiquiátrica en Argentina. El campo de la psiquiatría argentina finisecular estuvo marcado por intenciones de cambios y modernización de sus instituciones. En 1892, la dirección del hospicio recayó sobre Domingo Cabred, en cuyas propuestas para la institución destacó la integridad física y moral de los pacientes. Trabajó en el hospital por 40 años y, desde practicante a director de la institución, se desempeñó en diversos cargos. Hugo Vezzetti sostiene que la tarea de Cabred como psiquiatra en el ámbito público -pero también como sanitarista y reformador de la medicina pública- contribuyó en jerarquizar a la disciplina dentro de la medicina, en un momento en que las patologías mentales comenzaban a cobrar gran relevancia y reconocimiento en todo el ámbito nacional. Su proyecto modernizador combinaba así la organización médica de la asistencia -con salas de clinoterapia, médicos internos, vigilancia continua, etc.- con una organización de los pacientes alrededor del trabajo en talleres, con la recepción de un peculio (Vezzetti, 1991, p. 59).

La construcción del laboratorio de anatomía patológica que estudiamos en este artículo se realizó dentro de este marco de modernización de la institución hospitalaria, donde también Cabred creó, por ejemplo, el Instituto de Clínica Psiquiátrica. Al igual que la mayoría de los psiquiatras destacados de la época que realizaban viajes al viejo continente para conocer cómo se trabajaba en la disciplina psiquiátrica, el director del hospital se inspiró para sus proyectos institucionales en experiencias europeas. En 1896, viajó a Francia, Alemania y Bélgica para investigar la organización de los asilos europeos. En Eriangen, Alemania, el medico argentino contrató a Cristofredo Jacob para fundar el Laboratorio de Anatomía Patológica en Las Mercedes con el "fin de correlacionar los síntomas que los pacientes presentaban en vida con la lesión orgánica determinante, que se encontraría en la autopsia" (Volmer, 2010, p. 57). Para Cabred, la práctica de la autopsia iluminaba la relación entre el cuerpo y las enfermedades mentales. En el discurso que brindó para la inauguración de dicho laboratorio, en 1920, Cabred expresaba, "con [autopsia,] se descubren alteraciones dignas de tenerse en cuenta en la patogenia y en la terapéutica de estas enfermedades". ${ }^{4}$ De su trabajo, Vezzetti destaca la combinación del abordaje neuropsiquiátrico y los presupuestos sociológicos naturalistas (refiriéndose a la experiencia terapéutica del trabajo) como dos lógicas de abordaje de la locura (Vezzetti, 1991, p. 60). 
Como ya mencionamos, el doctor Jacob estuvo a cargo del Laboratorio de Anatomía Patológica, donde se desempeñó entre 1899 y 1911 . Dicho laboratorio se construyó con dinero de la Municipalidad de Buenos Aires. Contaba con una Sala de Microscopía, depósito de cadáveres y museo de piezas anatomopatológicas. También se construyeron salas de macro y microfotografía, salas de química biológica y de psicología experimental, sala de fonografía, una con aparatos antropométricos y otra con aparatos eléctricos, como la rueda de Wimshurst, para tratar condiciones tales como la parálisis de pares craneanos y el dolor de cabeza (Volmer, 2010, p. 59).

Entre los años 1901 y 1918, se hicieron 221 autopsias en total en el Laboratorio de Anatomía Patológica del Hospicio de las Mercedes. ${ }^{5}$ Dicho laboratorio fue importante para la producción de conocimiento psiquiátrico y la formación de estudiantes de medicina, quienes, en esa época, asistieron al laboratorio y presenciaron o realizaron autopsias de los pacientes. Para Cabred, la técnica de autopsia enseñaba a los estudiantes la conexión entre el cuerpo anatómico y las psicopatologías. En ese periodo, los estudiantes realizaron para sus tesis, trabajos inspirados en método anatomo-clínico, como expresaba el reconocido médico en 1920 (Golcman, 2015b, p. 168). ${ }^{6}$

En el Registro de Autopsias, los médicos consignaban datos personales del paciente internado, incluyendo su nombre, edad, fechas de admisión y muerte, ocupación anterior y "forma de psicopatía" (Véase cuadro № 1). Los sumarios generales para cada autopsia consistían en una descripción de la causa de muerte, y observaciones microscópicas del cerebro y demás órganos. Específicamente sobre las autopsias, los médicos escribieron en el registro datos como los siguientes:

- Aspecto externo: estado de nutrición; rigidez post-mortem; señales exteriores, excoriaciones, escaras, lesiones o signos de la enfermedad.

- Cabeza: cráneo; oído y ojos; dura madre; espacio subdural; piamadre y aracnoides; espacio subaracnoideo; vasos.

- Encéfalo: aspecto general, vascularización.

- Cerebro: hemisferios; circunvoluciones; corteza; sustancia blanca; ventrículos; glándula pineal y cuerpo pituitario; cuerpo estriado; talamos ópticos; cuerpos cuadrigéminos; cuerpo calloso y trígono cerebral; cerebelo; protuberancia anular; bulbo.
- Medula espinal, Meninges: gran simpático, ganglios, nervios periféricos.

- Cuello: cuerpo tiroideos y ganglios cervicales; nariz, laringe, tráquea; lengua, faringe, esófago.

- Tórax: costillas; pleura; bronquios; ganglios bronquiales; pulmones; corazón; pericardio; endocardio; válvula pulmonar, válvula aortita, válvula mitral; miocardio; arterias coronarias; aorta; estado de la sangre.

- Abdomen: peritoneo; hígado; estomago, vesículas; baz; riñones; uréteres; capsulas suprarrenales; páncreas; ganglios intestino delgado, intestino grueso, apéndice vermicular; vejiga. ${ }^{7}$

Para cada órgano mencionado, precisaban su peso, tamaño, vascularización, simetría, congestión, adherencias, entre otras características. Por ejemplo, el registro indica que un agricultor en Buenos Aires, el paciente $P C$, había llegado al hospital en octubre del año 1910 , cuando fue diagnosticado con psicopatía del "delirio toxico (uremia)"; y que murió en el mismo mes por "nefritis crónica". Su encéfalo se caracterizó por "congestión." Sus riñones eran "chicos" con "cortezas disminuidas" y también "congestionados". ${ }^{8}$

En el cuadro 1, mostramos el Registro de Autopsias entre los años 1901 y 1918, para conocer las características demográficas de pacientes que murieron en el Hospicio de las Mercedes en esa época.

La población urbana en el cambio de siglo en Buenos Aires recibía servicios psiquiátricos a través diversas instituciones que dependían de comunidades de inmigrantes extranjeros, de la Sociedad de Beneficencia y del gobierno municipal. Los cinco hospitales de las comunidades extranjeras en la capital eran el Hospital Francés, fundado por la Société Philantropique Française, el Hospital Británico, el Hospital Español, el Hospital Italiano y el Hospital Alemán. Los hospitales del gobierno municipal fueron el Hospital General de Hombres, el Hospital San Roque, el Hospicio de Mujeres Dementes, el Hospital General de Mujeres, el Hospital de Niños, el Hospital Militar y la institución de la que nos ocupamos, el Hospicio de las Mercedes (Santi, 2006).

Los hospitales dependientes del gobierno atendían principalmente a pacientes con un nivel social y económico menor que el de los que asistían a los hospitales de comunidades extranjeras mencionados arriba. El Registro de Autopsias muestra algunos datos socioeconómicos de los pacientes que murieron en el Hospicio de las Mercedes en esa época, que, 
Cuadro 1. Características demográficas de pacientes autopsiados entre los años 1901 y 1918 en el Hospicio de las Mercedes ${ }^{9}$

\begin{tabular}{|c|c|}
\hline Edad mediana & $38(6-70)$ \\
\hline Ocupación anterior a la admisión & $\begin{array}{l}\text { Número de } \\
\text { pacientes }\end{array}$ \\
\hline Jornalero & 36 \\
\hline Agricultor & 10 \\
\hline Cocinero & 6 \\
\hline Empleado & 5 \\
\hline Zapatero & 5 \\
\hline Carpintero & 4 \\
\hline Panadero & 4 \\
\hline Carnicero & 3 \\
\hline Sastre & 3 \\
\hline Albañil & 2 \\
\hline Contador & 2 \\
\hline Herrero & 2 \\
\hline Topógrafo & 2 \\
\hline Cañero & 1 \\
\hline Carrero & 1 \\
\hline Chacarero & 1 \\
\hline Cuarteador & 1 \\
\hline Hornero & 1 \\
\hline Maquinista & 1 \\
\hline Marinero & 1 \\
\hline Militar & 1 \\
\hline Músico & 1 \\
\hline Peluquero & 1 \\
\hline Pintor & 1 \\
\hline Relojero & 1 \\
\hline Sombrerero & 1 \\
\hline Talabartero & 1 \\
\hline Ninguna & 5 \\
\hline
\end{tabular}

en general, pertenecían a la creciente clase obrera. Como puede verse en el cuadro $\mathrm{N}^{\circ} 1$, la "ocupación anterior de admisión" para la mayoría de ellos fue la de jornalero. También encontramos agricultores, cocineros, zapateros y empleados. La historiadora Isabel Santi ha demostrado que muchos de estos pacientes de la clase obrera fueron inmigrantes europeos, especialmente de Italia, pero también de España, Francia e Irlanda (Santi, 2006). En el Registro de Autopsia no figuran datos del país de origen específicamente, pero gran parte de los apellidos de pacientes autopsiados allí son de origen italiano (como Crespo, Berlusconi, Manetti). La edad mediana de muerte de los pacien- tes en este hospital fue de 36 años, ${ }^{10}$ que se acerca a la expectativa de vida promedio en 1900 en América Latina, de 29 años. De acuerdo con este dato, podemos señalar que quienes habitaban la institución no tuvieron una vida particularmente corta (Pan American Health Organization, 2012).

La prevalencia de los inmigrantes en Las Mercedes reflejó, en general, las características demográficas del país para las primeras décadas del siglo XX. Entre 1881 y 1914 llegaron 4.200.000 personas a la Argentina. Entre esos inmigrantes, alrededor de 2.000 .000 eran italianos, 1.4000 .000 españoles y 170.000 franceses. Según el censo de 1895, un tercio de los extranjeros que llegaron a Argentina vivieron en Buenos Aires; al mismo tiempo, el $81 \%$ de los trabajadores en la capital eran extranjeros. ${ }^{11}$ Esta inmigración masiva de hombres europeos de bajo nivel socioeconómico y generalmente sin familias creó una nueva clase media urbana que amenazó la autoridad de las élites terratenientes en el cambio de siglo. Además, ciertas instituciones -como la Policía en Buenos Aires, o los hospitales psiquiátricos- eran espacios que contribuían en la adaptación de inmigrantes mediante la cual se pretendía llegar en la capital al orden deseado por las élites del Estado (Vezzetti, 1996). El discurso psiquiátrico de la Argentina finisecular entendía a los inmigrantes como una población patológica vinculada a desviaciones sexuales (por ejemplo, inversión, homosexualidad) y valores políticos amorales (por ejemplo, anarquismo, militancia gremial), que interrumpieron el "equilibrio higiénico" de la sociedad (Salessi, 1995).

Nuevas investigaciones han demostrado que la alta presencia de extranjeros en los hospitales psiquiátricos pudo haber tenido causas no relacionadas con cuadros patológicos determinados. Así, una de las autoras de este trabajo (AG) sostiene para el Hospital Esteves de Lomas de Zamora (anexo del Hospital Nacional de Alienadas), que es probable que la falta de un hogar propio, los bajos recursos de los recién llegados, las carencias afectivas familiares y amicales, hayan sido todas causas que influyeron en las mujeres extranjeras admitidas en dicha institución. Esto habría implicado que, en ocasiones, las mujeres fueran internadas por el hecho de estar atravesando situaciones emocionales difíciles y no necesariamente enfermedades psiquiátricas, pero que una vez internadas en el hospital, sufrieran un proceso de cronificación (Golcman, 2015a, p. 125; Ríos Molina, 2009, p. 44). Además, se manejan ciertos datos que permiten hablar de una sobrerrepresentación de extranjeras "locas" en la institución; por ejemplo, el censo 
de 1914, que recogió también información sobre las "idiotas" y las "alienadas", tanto entre las argentinas como entre las extranjeras. Se entiende que los datos que ofrece el censo pertenecen a la población fuera de las instituciones; en esta población censada hay más argentinas que extranjeras (65\% idiotas y $54 \%$ alienadas) (Golcman, 2015a, pp. 125-126).

En el cuadro 2, describimos la práctica de autopsia y el marco diagnóstico científico que caracterizó a estos pacientes extranjeros, percibidos como un peligro a la cultura nacional, en Las Mercedes.

Cuadro 2. Autopsias y diagnósticos de psicopatía entre los años 1901 y 1918 en el Hospicio de las Mercedes ${ }^{12}$

\begin{tabular}{|c|c|}
\hline Diagnóstico & $\begin{array}{c}\text { Autopsias } \\
\text { (Total = 221) }\end{array}$ \\
\hline Parálisis general progresiva & $44(20 \%)$ \\
\hline Demencia & $42(19 \%)$ \\
\hline Precoz & 14 \\
\hline Vesánica & 7 \\
\hline Senil & 5 \\
\hline Alcohólico & 5 \\
\hline Orgánica & 4 \\
\hline Epiléptica & 3 \\
\hline Alcoholismo & $26(12 \%)$ \\
\hline Subagudo & 7 \\
\hline Crónico & 6 \\
\hline Con ideas de persecución & 6 \\
\hline Agudo & 1 \\
\hline Delirio & $21(10 \%)$ \\
\hline Agudo & 5 \\
\hline Infeccioso & 3 \\
\hline Persecutorio & 3 \\
\hline Alcohólico & 3 \\
\hline Sistematizado progresivo & 3 \\
\hline Tóxico & 1 \\
\hline Manía & $11(5 \%)$ \\
\hline Alcohólica & 8 \\
\hline Aguda & 2 \\
\hline Melancolía & $10(5 \%)$ \\
\hline Alcohólica & 5 \\
\hline Apática & 2 \\
\hline Epilepsia & $10(5 \%)$ \\
\hline Cretinismo & $1(<1 \%)$ \\
\hline Monomanía homocido & $1(<1 \%)$ \\
\hline Sin diagnóstico & $56(25 \%)$ \\
\hline
\end{tabular}

En Las Mercedes, la categoría de demencia constituyó el $19 \%$ de los diagnósticos en esa época, y el tipo de demencia más común entre los pacientes autopsiados. Basado en la teoría del psiquiatra alemán Emil Kraepelin, la demencia precoz se definió como una psicosis con deterioro temprano y permanente de la capacidad cognitiva (Golcman, 2015a, p. 160; Makari, 2012). En un trabajo anterior, Golcman ha sostenido que debates sobre demencia precoz y esquizofrenia, basados en lecturas locales de teorías europeas, fueron centrales de la formación de la profesión psiquiátrica argentina en los principios del siglo XX (Golcman, 2015b). La esquizofrenia, por su parte, fue presentada en 1911 por Eugene Bleuler, y para gran parte de la psiquiatría fue una versión más compleja y evolucionada del diagnóstico de demencia precoz. En nuestro país encontramos artículos científicos sobre esta patología desde 1903 hasta 1943, y sobre esquizofrenia, desde 1920 (Golcman, 2015b).

Además de demencia precoz, en la categoría de demencia se incluyó la demencia vesánica y la senil -que constituyeron siete y cinco casos, respectivamente- y las demencias definidas por sus causas orgánicas, tales como "alcohólico", "epiléptico" o simplemente "orgánico". El énfasis en las causas orgánicas era una característica general de la clasificación de las enfermedades mentales en el Laboratorio de Autopsia del Hospicio de las Mercedes entre 1901 y 1918. Por ejemplo, el diagnóstico más común, Parálisis general progresiva, se definió implícitamente por su causa corporal, es decir, por la progresión del neurosífilis. Otros diagnósticos, "delirio," por ejemplo, tenían sus raíces en infecciones o causas tóxicas. La centralidad de la etiología corporal de las enfermedades mentales en la práctica clínica en Las Mercedes era evidente en otros documentos, como el "Boletín Anamnésico". Según el análisis histórico de Navarlaz y Miranda, en este Boletín, entre 1900 y 1930, los médicos registraron los datos de los pacientes al ingresar a Las Mercedes (Navarlaz y Miranda, 2009). Uno de los ítems consignados allí era: “¿Cuál es la causa probable de la enfermedad?". Como expresan Navarlaz y Miranda, esta pregunta demostraba la importancia de las causas orgánicas en la práctica psiquiátrica en Las Mercedes (Navarlaz y Miranda, 2009). Los médicos compararían y contrastarían las observaciones iniciales y tentativas sobre las causas orgánicas al ingreso -expresadas en el boletín- con la certeza de las observaciones directas del cuerpo autopsiado post-mortem también descriptas en el registro.

Por otra parte, el alcohol, en particular, por sus vínculos a la figura de delincuente inmigrante, fue considera- 
do una causa orgánica de diversas psicopatologías en Las Mercedes, entre las que se incluyeron demencia, delirio, manía, melancolía y por supuesto, alcoholismo. En total, el $22 \%$ de los diagnósticos de psicopatía tuvieron relación con el alcohol. ${ }^{13}$ En el hospicio, los médicos entendieron, al mismo tiempo, el alcohol como una causa tóxica de las enfermedades mentales (con otros tóxicos como el mercurio, el fósforo, etc.) y un estado patológico por sí mismo con etapas distintas (por ejemplo, agudo, subagudo, crónico) e ideas asociadas (por ejemplo, alcoholismo con ideas de persecución). Encontramos también en el Boletín Anamnésico mencionado, en la parte superior, la pregunta de admisión a la institución: “¿Ha abusado de bebidas alcohólicas?”.

Para el director Cabred, las prácticas del laboratorio -no sólo la autopsia, sino también los análisis de sangre- eran tecnologías críticas para sostener este marco diagnostico higiénico en el hospital. Al menos en principio, las autopsias demostraron la conexión causal entre los cambios del cuerpo anatomoclínico y la psicopatología; de este modo establecía la disciplina psiquiátrica como una rama legítima de la medicina científica. Al ingreso del paciente en el Hospicio de las Mercedes, los médicos analizaron el cuerpo vivo a través de las observaciones clínicas de los signos y síntomas. Después de muerto, la anatomía patológica confirmaba (o no) lo observado en el ingreso, y precisaba el diagnóstico del paciente por la investigación directa de las lesiones corporales que causaban la locura. De hecho, el registro diferenciaba la "Forma de psicopatía en el momento del ingreso", basado en las observaciones clínicas, con la "Forma de psicopatía en el momento de la muerte", a partir de la autopsia. Así se facilitaba la comparación entre diagnósticos fundados en la clínica en contraposición con las observaciones hechas en la sala de autopsias. ${ }^{14}$

En el Congreso Internacional de Medicina Mental de París de 1889, Cabred reveló que esta perspectiva anatomoclínica e higiénica que caracterizaba a la práctica de autopsias en el hospicio tenía sus raíces en la teoría de degeneración del psiquiatra francés Bénédict Morel. El degeneracionismo fue una teoría psiquiátrica desarrollada por Morel en 1857, en su trabajo Traité des dégénérescences physiques, intellectuelles et morales de l'espèce humaine et des causes qui produisent ces variétés maladives; y posteriormente reinterpretada por Valentin Magnan. Para Morel, los trastornos psíquicos eran una expresión de la constitución anormal de los organismos de los sujetos que los presentaban. Este proceso de degeneración tenía un carácter anatómico, donde la hipertrofia o la atrofia de ciertos órganos se- ñalaban el nivel del desarrollo evolutivo del sujeto. ${ }^{15}$ Por ejemplo, como comentan Navarlaz y Miranda, el médico argentino Francisco de Veyga escribió en su libro Degeneración y degenerados:

...Bourneville, separa entre los idiotas y cretinos, estados donde al lado de un estado mental de la más extraña degradación, se muestra un estado físico caracterizado por la hinchazón general sobre todo del cuello y las extremidades. Es el mixedema, el cretinismo mixedematoso. ${ }^{16}$

Eran deberes del médico vigilar y guardar por el bien social; de este modo, se generaba una explicación científica que daba respuestas a una problemática social (Golcman, 2015a, p. 123; Peset y Huertas, 1986).

Uno de los estereotipos de degeneración en el ámbito psiquiátrico argentino fue el inmigrante italiano. En el Hospicio de las Mercedes, el director Lucio Meléndez (que asumió su cargo en 1876 y comenzó una publicación en la Revista Médico Quirúrgica, donde se trató esta temática), y su sucesor Cabred teorizaron el "loco inmigrante". En palabras de Meléndez, en el Informe del Hospicio en 1885,

Como ya he dicho en varias ocasiones los inmigrantes italianos son los que dan mayor cantidad de locos, no siendo infrecuente notar, que al llegar a este país vienen ya locos, sordo-mudos o defectuosos, por lo que debo suponer se embarcaran así en el país de su procedencia. En estos europeos que he tenido también ocasión de comprobar la existencia de la predisposición hereditaria, tanto por la rama materna como por la paterna. ${ }^{17}$

Para Meléndez, ese "inmigrante italiano" constituía una figura viciosa, y con frecuencia alcohólica, que debía estar bajo la autoridad del sistema nacional psiquiátrico.

Como ya hemos discutido, el alcohol fue una causa principal de degeneración según los médicos positivistas, especialmente en la población de trabajadores italianos en Buenos Aires (Rossi, 2006). Morel y otros hipotetizaron que la intoxicación alcohólica (entre otras intoxicaciones) tenía efectos sustantivos en los órganos, no sólo de los degenerados, sino también sus niños. Como De Veyga expresaba,

...los niños nacidos bajo la influencia del alcoholismo de los padres, sufren las consecuencias del estado convulsivo seguido de estupor que determina el alcohol en los que abusan de este: la histeria, la epilepsia, la imbecilidad... Las tendencias instintivas más perversas se ven en los hijos de alcoholistas tal cual existen momentáneamente en los degenerados. ${ }^{18}$ 
La otra causa orgánica de la degeneración era la sífilis, y su consecuencia más directa, la parálisis general progresiva. Este diagnóstico era lo más común en Las Mercedes entre los años 1901 a 1918, y un veinte por ciento de los pacientes autopsiados lo padecieron. En Argentina, a partir de 1920, sífilis y alcoholismo como causas directas de degeneración-fueron objeto de campañas masivas de salud realizadas por la Liga Argentina de Higiene Mental, una organización fundada por el futuro director del Hospicio de las Mercedes, el psiquiatra Gonzalo Bosch (quien asumió la dirección en 1931) (Navarlaz y Miranda, 2009).

\section{CONCLUSIÓN}

El trabajo en el laboratorio de anatomía patológica practicado en el Hospicio de las Mercedes fue una muestra de un tipo de investigación psiquiátrica que se hacía en todo el mundo y que se reprodujo en Argentina. En ese espacio se pretendió trabajar del mismo modo que se hacía en Europa, por lo que se puso a cargo de él a un europeo, y hasta se copió el modelo de laboratorio. Esta experiencia de trabajo muestra cómo circularon ciertas prácticas y tecnologías psi-

\section{NOTAS}

1. Desde la historia de la psiquiatría, podemos ubicar la anatomía patológica en lo que se llamó el modelo moderno de enfermedad (entre finales del siglo XIX e inicios del XX). A partir del mismo, se relacionó la patología con un órgano, lo que significó la entronización del cerebro (Golcman, 2015a). Desde este modelo se buscaba delimitar en un paciente la presencia de ciertos elementos pertenecientes a la semiología psiquiátrica, y no se intentaba conocerlo en cuanto sujeto (Lantéri-Laura, 2000, p. 173)

2. Como explica Carlos Stagnaro, la historia clásica de la psiquiatría en nuestro país hizo hincapié en los trabajos sobre los personajes importantes dentro del campo, aquellos que fueron marcando épocas y marcaron hitos relevantes en el devenir de la profesión (Stagnaro, 2006, pp. 7-39).

3. Para más sobre la figura de Jacob y el desarrollo de la anatomía patológica en la Argentina véase (Guerrino, 1982; Loudet y Loudet (h), 1971). Para una biografía completa de Jacob véanse los textos de dos de sus discípulos, (López Pasquali, 1965 y Orlando, 1996).

4. Cabred, Domingo (1920a), "Discurso introduciendo los pabellones de clinoterapia y del laboratorio de la clínica de psiquiatría", Buenos Aires, p. 11.

5. Cabred, Domingo (1902), Registro de Autopsias de las Mercedes, Buenos Aires, p. 2. quiátricas desde el viejo continente, y cómo se reinterpretaron y amoldaron al contexto nacional.

La información que brindan los registros del laboratorio del hospicio muestra algunos datos de la población de la institución, como las profesiones y las nacionalidades de los pacientes; además, se numeran los diagnósticos utilizados por los médicos. Estos datos, más que permitirnos conocer a los pacientes, brindan la posibilidad de entender el modo en que estos fueron analizados por los psiquiatras. En este contexto, claramente el desarrollo de la anatomía patológica era más relevante como un espacio de investigación que para el tratamiento concreto de los pacientes, al menos en el corto plazo, ya que se trataba de una actividad practicada postmortem. De igual modo, esta disciplina implicaba ubicar al Hospicio de las Mercedes (y, por lo tanto, al Estado argentino) a la altura de los hospitales más destacados a nivel mundial. Los médicos que trabajaban en él figuraban como verdaderos representantes de la psiquiatría: profesionales serios que participaron en el devenir de una disciplina en un proceso de especialización -que se extendió hasta la segunda mitad del siglo XX- y del camino por encontrar su lugar dentro del campo médico.

6. Véase Cabred, Domingo (1920b), "Antecedentes de la fundación del asilo-colonia de Lujan", Revista de Psiquiatría, Criminología y Medicina Legal, Vol. 1, Buenos Aires, pp. 5-7..

7. Véase también el análisis de esas fuentes en (Navarlaz y Miranda, 2009, p. 16).

8. Cabred, Domingo (1902), Registro de Autopsias de las Mercedes, Buenos Aires, p. 110.

9. Cabred, Domingo (1902), Registro de Autopsias de las Mercedes, Buenos Aires.

10. Cabred, Domingo (1902), Registro de Autopsias de las Mercedes, Buenos Aires.

11. Esas figuras son de (Santi, 2006).

12. Cabred, Domingo (1902), Registro de Autopsias de las Mercedes, Buenos Aires.

13. Cabred, Domingo (1902), Registro de Autopsias de las Mercedes, Buenos Aires.

14. Cabred, Domingo (1902), Registro de Autopsias de las Mercedes, Buenos Aires.

15. Senet, R. (1906), "Los estigmas somáticos de degeneración y la filogenia", Archivos de Psiquiatría y Criminología aplicadas a las ciencias afines, 5, pp. 549-581. 
16. De Veyga, Francisco (1938), Degeneración y degenerados, Buenos Aires, Librería y Editorial El Ateneo, citado en (Navarlaz, 2009).

17. Meléndez, Lucio (1886) "Tumor intracraneano sifilítico," Revista Médico Quirúrgica, XIX, p. 354-358, citado por (Santi, 2006).

\section{BIBLIOGRAFÍA}

Ablard, Jonathan (2008), Madness in Buenos Aires. Patients, Psychiatrists and the Argentine State, 1880-1983, Canadá, University of Calgary Press.

Bohoslavsky, Ernesto Lázaro (2005), "Sobre los límites del control social. Estado, historia y política en la periferia argentina (1890-1930)". En: Di Liscia, María Silvia; Bohoslavsky, Ernesto Lázaro (eds.), Instituciones y formas de control social en América Latina, 1840-1940. Una revisión, Buenos Aires, Prometeo, pp. 49-50.

Golcman, Aida Alejandra (2015a), "Interpretación de la locura y acciones concretas en el Hospital José A. Esteves, de Lomas de Zamora (1908-1971)", Tesis de Doctorado, IDES-UNGS, Inédita.

Golcman, Aida Alejandra (2015b), "El diagnóstico de la demencia precoz y la esquizofrenia en Argentina, 1920-1940", Trashumante: Revista Americana de Historia Social, 5, pp. 150-172.

Guerrino, Antonio Alberto (1982), La psiquiatría argentina, Buenos Aires, Ediciones Cuatro.

Lantéri-Laura, Georges (2000), Ensayo sobre los paradigmas de la psiquiatría moderna, Madrid, Tricastela.

López Pasquali, L. (1965), Christfried Jacob, su obra neurológica, su pensamiento psicológico y filosófico, Buenos Aires, López Libreros Editores, Talleres Gráficos de La Prensa Médica Argentina.

Loudet, Osvaldo y Loudet, Osvaldo (hijo) (1971), Historia de la psiquiatría argentina, Buenos Aires, Troquel.

Makari, George (2012), Revolución en mente. La creación del psicoanálisis, México, Sexto Piso.

Navarlaz, Vanesa Eval y Miranda, Mariela Fabiana (2009), "La hipótesis de la degeneración en las historias clínicas del Hospicio de las Mercedes y la Colonia Dr. Cabred entre los años 1900 y 1930", Anuario de investigaciones de Facultad de Psicología de Universidad de Buenos Aires, XVI, pp. 183-191.

Orlando, Jacinto Carlos (1996), Christofredo Jacob, su vida y obra (1866-1946), Buenos Aires, Mundi.

Pan American Health Organization (2012), "Latin America and the Caribbean Have Gained 45 years in Life Expectancy Since 1900" [en línea], disponible en: http://goo.gl/Fvez6Q [consultado el 7/4/2017].
18. De Veyga, Francisco, (1938) Degeneración y degenerados, Buenos Aires, Librería y Editorial El Ateneo, citado en (Navarlaz, 2009).

Peset, José Luis y Huertas, Rafael (1986), “Del ángel caído al enfermo mental: sobre el concepto de degeneración en las obras de Morel y Magnan", Asclepio, 3(8), pp. 215-240.

Ríos Molina, Andrés (2009), La locura durante la Revolución mexicana. Los primeros años del Manicomio General La Castañeda, 1910-1920, México, El Colegio de México.

Roig, Arturo (1963), "Notas sobre el eclecticismo en Argentina," Revista de Historia Americana y Argentina, V-VI, pp. 159-182.

Rossi, Lucia, (2006), "Argentina: profilaxis social en la década del 20," Revista de Historia de la Psicología, 27 (1), pp. 95-108.

Salessi, Jorge (1995), Médicos maleantes y maricas: higiene, criminología y homosexualidad en la construcción de la nación argentina (Buenos Aires, 1871-1914), Buenos Aires, B. Viterbo.

Santi, Isabel (2006), "El paciente inmigrante en Buenos Aires a fines del siglo XIX. De la filantropía a la tecnología alienista", Amérique Latine Histoire et Mémoire. Les Cahiers $A L H I M, 12$, [en línea], disponible en: https://alhim.revues. org/1322 [consultado el 7/4/2017].

Saurí, Jorge (1996), Historia de las ideas psiquiátricas. El naturalismo psiquiátrico, Buenos Aires, Lohlé-Lumen.

Stagnaro, Juan Carlos (2006), “Evolución y situación actual de la historiografía de la psiquiatría en la Argentina", Frenia. Revista de Historia de la Psiquiatría, VI, pp. 7-39.

Talak, Ana María (2005), “Eugenesia e higiene mental: usos de la psicología en la Argentina, 1900-1940". En: Miranda, Marisa; Vallejo, Gustavo (eds.), Darwinismo social y eugenesia en el mundo latino, Buenos Aires, Siglo XXI, pp. 563-599.

Terán, Oscar (1987), Positivismo y nación en la Argentina, Buenos Aires, Puntosur.

Terán, Oscar (2000), "El pensamiento finisecular (1880-1916)". En: Lobato, Mirta (ed.), El progreso, la modernización y sus límites (1880-1916), Buenos Aires, Sudamericana, V, pp. 327-364.

Terán, Oscar (2004), “Ideas e intelectuales en la Argentina, 1880-1980". En: Terán, Oscar (ed.), Ideas en el siglo: Intelectuales y cultura en el siglo XX latinoamericana, Buenos Aires, Siglo XXI, pp. 13-97. 
Vezzetti, Hugo (1985), “El discurso psiquiátrico”. En: Biagini, Hugo (comp.), El Movimiento positivista argentino, Buenos Aires, Editorial de Belgrano.

Vezzetti, Hugo (1991), "Domingo Cabred y el asilo de puertas abiertas", Vertex, Revista Argentina de Psiquiatría, 2 (3), p. 59.
Vezzetti, Hugo (1996), Aventuras de Freud en el país de los argentinos. De José Ingenieros a Enrique Pichon Rivière, Buenos Aires, Paidós.

Volmer, María Cristina (2010), Historia del Hospital Neuropsiquiátrico José T. Borda, Buenos Aires, Salerno. 